\title{
Prévalence de la cysticercose porcine dans le Mayo-Danay (Nord Cameroun) et le Mayo-Kebbi (sud-ouest du Tchad)
}

\author{
E. Assana ${ }^{1}$ P.A. Zoli ${ }^{1}$ H.A. Sadou ${ }^{2}$ \\ Nguekam $^{1}$ L. Vondou ${ }^{1}$ M.S.R. Pouedet ${ }^{1}$ \\ P. Dorny ${ }^{3}$ J. Brandt ${ }^{3}$ S. Geerts ${ }^{3 *}$
}

Mots-clés

Porcin - Taenia solium - Cysticercose Cameroun - Tchad.

\begin{abstract}
Résumé
Une étude menée de mai à octobre 1999 dans le département du MayoDanay (extrême nord du Cameroun) et dans la préfecture du Mayo-Kebbi (sud-ouest du Tchad) a eu pour but de déterminer la prévalence de la cysticercose porcine et d'identifier les principaux facteurs qui la favorisaient. Elle a montré que les conditions hygiéniques dans lesquelles vivait la population ainsi que celles de l'élevage des porcs étaient très médiocres, avec pour conséquence des infestations massives de la population porcine par des cysticerques de Taenia solium. En effet, 42 p. 100 des 126 exploitations visitées étaient dépourvues de latrines et les porcs, en divagation permanente ou semi-permanente, avaient facilement accès aux matières fécales humaines déposées aux alentours des habitations. Le diagnostic clinique effectué par la méthode du langueyage a montré que 20,5 p. 100 des 852 porcs vivants examinés étaient porteurs de cysticerques. L'inspection des carcasses réalisée dans les abattoirs locaux a révélé que 15,7 p. 100 des 51 porcs abattus étaient ladres. Parmi les 264 sérums de porcs soumis au test Elisa pour la détection des antigènes circulants de cysticerques, 105 (39,8 p. 100) se sont révélés positifs. Ces résultats ont indiqué que le Mayo-Danay et le Mayo-Kebbi étaient d'importants foyers de la cysticercose porcine à Taenia solium.
\end{abstract}

\section{INTRODUCTION}

La cysticercose, due aux larves de Taenia solium ou Cysticercus cellulosae, constitue un problème économique et de santé publique important qui est cependant relativement méconnu dans un grand nombre de pays africains $(6,7,17)$. Pourtant, à l'exception des régions où l'élevage et surtout la consommation du porc constituent un tabou religieux, la cysticercose porcine affecte probablement tous les pays au sud du Sahara où les conditions d'apparition et de perpétuation de cette zoonose sont généralement réunies : conditions hygiéniques pauvres, absence quasi généralisée de latrines, surtout dans les zones rurales, divagation permanente ou saisonnière des porcs et absence totale d'inspection de la viande

\footnotetext{
1. Université de Dschang, Cameroun

2. Délégation départementale de l'élevage, des pêches et des industries animales du Mayo-Danay à Yagoua, Cameroun

3. Institut de médecine tropicale, Anvers, Belgique

* Auteur pour la correspondance

Tél : +32 (0)324 76262 ; fax: +32 (0)32476268 ; e-mail : sgeerts@itg.be
}

des porcs (16). Contrairement à l'Amérique latine, les données sur la cysticercose porcine sont cependant plutôt rares en Afrique bien que quelques études y aient été menées. Chambers (4) rapporte qu'au Zimbabwe 67,6 p. 100 des carcasses de porcs saisies en un an dans les abattoirs étaient ladres. Au Nigeria une prévalence de 20 p. 100 a été rapportée dans l'Etat d'Enugu (14). En Tanzanie un taux moyen d'infestation de 13,3 p. 100 a été déterminé dans trois communes du pays (2). Au Cameroun la fréquence et la distribution de la cysticercose porcine sont également assez mal connues. Les études menées jusqu'ici sur cette maladie ont été focalisées surtout dans la région de l'Ouest $(9,10,12,15,18,19)$. Au nord du pays, Awa et coll. (1) ont rapporté que 12,3 p. 100 des 750 carcasses inspectées à l'abattoir de Garoua étaient infestées. Au Tchad, Graber et Chailloux (8) ont rapporté des cas de ladrerie massive chez des porcs abattus à Fort-Lamy. En dehors de ces travaux, il n'existe pas d'autres données sur la cysticercose porcine et humaine dans la partie septentrionale du Cameroun et au Tchad, surtout dans les grandes régions d'élevage porcin de l'extrême nord du Cameroun et du sud-ouest du Tchad (13). C'est pour cela que les auteurs ont entrepris une étude qui a eu pour objectifs d'identifier les facteurs susceptibles de favoriser l'infestation des porcs par les œufs de $T$. solium et de déterminer la prévalence de 
la zoonose. Les diagnostics ont été réalisés sur les animaux vivants et abattus et également par l'examen sérologique grâce au test Elisa-sandwich pour la détection des antigènes circulants des métacestodes du Taenia spp.

\section{MATERIEL ET METHODES}

L'enquête a eu lieu, d'une part, sur deux marchés du département du Mayo-Danay (extême nord du Cameroun) situés à une dizaine de kilomètres chacun de la frontière entre le Tchad et le Cameroun et, d'autre part, dans 126 élevages dont 70 dans le département du Mayo-Danay et 56 dans la préfecture du Mayo-Kebbi (sud-ouest du Tchad). Ces deux régions d'étude s'étendent entre $9^{\circ}$ et $11^{\circ} 15^{\prime}$ de lat. N., et $14^{\circ}$ et $16^{\circ} 30^{\prime}$ de long. E. Les exploitations, choisies au hasard, abritaient un total de 2065 porcs. Un questionnaire a servi pour la collecte des données sur le système d'élevage, les conditions hygiéniques et sanitaires dans les exploitations, le niveau de connaissance des éleveurs par rapport au complexe taeniose-cysticercose. Des observations directes sur l'environnement des exploitations ont permis également de déterminer les habitudes hygiéniques des éleveurs ainsi que celles de leurs voisins non éleveurs, mais pouvant avoir des contacts, d'une façon ou d'une autre, avec les porcs.

\section{Animaux}

Un total de 903 porcs de races locales dont 852 vivants (566 adultes de plus d'un an et 286 jeunes de moins d'un an) et 51 abattus (23 de plus d'un an et 28 de moins d'un an) ont été examinés par la méthode du langueyage, pour les animaux vivants, et l'inspection des carcasses pour ceux qui avaient été abattus. Le langueyage consiste à examiner et/ou palper la face inférieure de la langue. L'inspection des carcasses a été effectuée sur les marchés par des infirmiers vétérinaires selon la méthode classique : des incisions ont été pratiquées au niveau des masséters, du cœur, des muscles fessiers, du diaphragme et de la langue.

\section{Sérums}

Des échantillons de sang ont été prélevés sur 255 des 852 porcs vivants qui n'ont pas révélé d'infestation lors de l'examen clinique et seulement sur 9 porcs abattus qui n'ont pas non plus révélé d'infestation lors des examens ante et post mortem. Les sérums ainsi obtenus ont été congelés à $-20^{\circ} \mathrm{C}$ jusqu' au moment de l'analyse.

\section{Elisa pour la détection d'antigènes circulants de cysticerques de Taenia solium}

L'Elisa-sandwich a été effectué selon Dorny et coll. (5). Les anticorps monoclonaux (Mab) B158C11A10 et B60H8A4 biotynilé ont été utilisés pour capturer les antigènes circulants. Ces MAb ont été développés contre les produits d'excrétion et de sécrétion de cysticerques de $T$. saginata, mais ils réagissent aussi bien avec ceux de T. solium (3). Le complexe streptavidine-péroxydase et l'orthophénylène diamine ont servi respectivement comme conjugué et comme chromogène. La réaction a été lue à l'aide d'un lecteur Elisa (Multiskan RC, Labsystems) à $492 \mathrm{~nm}$. Le seuil de positivité a été déterminé par comparaison de la densité optique (DO) de chaque échantillon avec la moyenne des DO d'une série de huit échantillons négatifs (provenant de porcs d'un élevage amélioré sans risque d'exposition aux œufs de T. solium), au seuil de probabilité de 0,001 (test de Student modifié).

\section{RESULTATS ET DISCUSSION}

\section{Système d'élevage porcin et conditions hygiéniques}

Les tableaux I à III résument les facteurs qui ont favorisé l'infestation des porcs par les métacestodes de T. solium. Ces facteurs ont été la divagation des animaux, le manque de latrines, les défécations à l'air libre à des endroits facilement accessibles aux porcs et la méconnaissance par la population du mode d'infestation et des aspects zoonotiques et pathologiques du parasite.

Bien que la majorité de la population musulmane du Cameroun se trouve dans les trois provinces septentrionales du pays (Adamaoua, Nord et Extrême Nord), elle y constitue cependant moins de la moitié de la population locale. Ce fait est encore plus marqué dans les provinces du Nord et de l'Extrême Nord où la majorité de la population est de religion chrétienne ou animiste. C'est la raison pour laquelle l'élevage du porc y est non seulement présent, mais il y prend depuis quelques années un développement toujours croissant. Les grandes métropoles de Yaoundé et de Douala sont régulièrement approvisionnées en porcs, davantage à partir de ces deux provinces que de celle de l'Ouest où le cheptel porcin est resté sérieusement réduit depuis le début des années 80 , suite à l'épizootie de peste porcine africaine (Ppa) enzootique dans la région (11). Les trois provinces du nord sont pour l'instant indemnes de la Ppa (1) et il est à souhaiter que la barrière sanitaire reste suffisamment étanche pour les préserver de cette épizootie. Toutefois, d'autres entraves à l'essor de l'élevage porcin existent dans le nord. Il s'agit notamment des pathologies parasitaires externes (poux, gales, etc.) et internes (helminthoses, etc.) et surtout nutritionnelles qui entraînent des mortalités de 30 à 42 p. 100 chez les porcelets avant l'âge d'un mois (1).

De plus, comme le montrent les tableaux I et II, les porcs sont élevés dans des conditions hygiéniques déplorables : 32,9 et 53,6 p. 100 des exploitations porcines visitées respectivement au Cameroun et au

\section{Tableau I}

Systèmes d'élevage dans les régions de l'enquête

\begin{tabular}{|c|c|c|c|c|c|c|c|c|c|c|c|}
\hline \multirow[t]{2}{*}{ Régions } & \multicolumn{2}{|c|}{$\begin{array}{l}\text { Elevages } \\
\text { enquêtés }\end{array}$} & \multicolumn{3}{|c|}{$\begin{array}{c}\text { Elevages } \\
\text { en divagation permanente }\end{array}$} & \multicolumn{3}{|c|}{$\begin{array}{c}\text { Elevages } \\
\text { en divagation saisonnière }\end{array}$} & \multicolumn{3}{|c|}{$\begin{array}{c}\text { Elevages } \\
\text { en claustration permanente }\end{array}$} \\
\hline & NEI & NPo & NEI & NPo & $\%$ El & NEI & NPo & $\%$ El & NEI & NPo & $\%$ El \\
\hline $\begin{array}{l}\text { Mayo-Danay } \\
\text { (Cameroun) }\end{array}$ & 70 & 1083 & 26 & 525 & 37,1 & 38 & 460 & 54,3 & 6 & 98 & 8,9 \\
\hline $\begin{array}{l}\text { Mayo-Kebbi } \\
\text { (Tchad) }\end{array}$ & 56 & 982 & 31 & 463 & 55,4 & 25 & 519 & 44,6 & 0 & 0 & 0 \\
\hline
\end{tabular}


Tableau II

Niveaux hygiéniques et sanitaires dans les exploitations porcines

\begin{tabular}{|c|c|c|c|c|}
\hline \multirow[t]{3}{*}{ Régions } & \multirow[t]{3}{*}{ Total exp. enquêtées } & \multirow[t]{2}{*}{ Exp. avec latrines } & \multicolumn{2}{|c|}{ Lieux de la défécation } \\
\hline & & & Porcherie & Autres * \\
\hline & & Nb. (\%) & Nb. & Nb. $(\%)$ \\
\hline $\begin{array}{l}\text { Mayo-Danay } \\
\text { ( Cameroun) }\end{array}$ & 70 & $57(67,1)$ & 0 & $23(32,9)$ \\
\hline $\begin{array}{l}\text { Mayo-Kebbi } \\
\text { (Tchad) }\end{array}$ & 56 & $26(46,4)$ & 0 & $30(53,6)$ \\
\hline
\end{tabular}

Exp : exploitations

* Champs, environs des habitations, touffes d'arbustes qui longent les pistes du bétail, etc.

\section{Tableau III}

Niveau de connaissance des éleveurs du complexe taeniose-cysticercose

\begin{tabular}{lcccc} 
Régions & $\begin{array}{c}\text { CExp porcines } \\
\text { enquêtées }\end{array}$ & $\begin{array}{c}\text { CExp connaissant } \\
\text { la C porcine }\end{array}$ & $\begin{array}{c}\text { CExp connaissant } \\
\text { la relation entre T et C }\end{array}$ & $\begin{array}{c}\text { CExp connaissant } \\
\text { la C humaine }\end{array}$ \\
\hline $\begin{array}{l}\text { Mayo-Danay } \\
\text { (Cameroun) }\end{array}$ & 70 & $54(77,1)$ & $\mathbf{N b .}(\%)$ & Nb. \\
$\begin{array}{l}\text { Mayo-Kebbi } \\
\text { (Tchad) }\end{array}$ & 56 & $31(55,4)$ & $7(12,9)$ & 0 \\
\end{tabular}

CExp : chefs d'exploitations ; $\mathrm{C}$ : cysticercose ; $\mathrm{T}$ : taeniose

Tchad étaient dépourvues de latrines. Même si, contrairement à ce qui se passe dans la province de l'Ouest (18), les porcheries ne servent pas de lieux de défécations, celles-ci se font le plus souvent à l'air libre dans des endroits facilement accessibles aux porcs dont à peu près 95 p. 100 sont en divagation permanente ou saisonnière (tableau I). Les centres urbains n'échappent pas non plus à cette règle dans la mesure où il y existe des terrains vagues qui servent de lieux de défécation et où les porcs, en divagation permanente, ont l'habitude de se rassembler.

Etant donné la structure des porcheries, généralement construites en matériaux locaux peu résistants, ou le fait que les porcs sont maintenus au piquet, il est possible à ces derniers de s'échapper facilement et de divaguer. Ils sont ainsi exposés à diverses infestations dont l'infestation par les œufs de T. solium contenus dans les matières fécales humaines déposées à l'air libre.

En ce qui concerne le niveau de connaissance des éleveurs au sujet du complexe taeniose-cysticercose, le tableau III montre qu'il y a encore un grand effort à faire dans le domaine de l'éducation sanitaire. Bien qu'environ deux tiers des chefs d'exploitation connaissaient la cysticercose porcine, il a semblé que très peu de gens se rendaient compte de sa relation avec la taeniose et la cysticercose humaine.

\section{Cysticercose porcine}

Les tableaux IV et $\mathrm{V}$ montrent les résultats du langueyage et de l'examen post mortem. En moyenne, 20,5 p. 100 des porcs vivants et 15,7 p. 100 des porcs abattus étaient ladres. Le nombre plus faible de porcs parasités observés lors de l'inspection de viande a été dû au fait que seuls les porcs qui ont été négatifs au langueyage ont été abattus. Ce chiffre de 15,7 p. 100 est deux fois supérieur à celui observé par Graber et Chailloux (8) dans la même région du Tchad et presque identique à celui d'Awa et coll. (1) dans le Nord Cameroun. En 1984-85, Zoli et coll. (18) ont trouvé une prévalence plus élevée (24,6 p. 100 au langueyage) dans le département de la Ménoua, même si Nguekam (12) et Pouedet (15), suite à une

\section{Tableau IV}

Prévalence de la cysticercose porcine dans les zones de l'étude (langueyage)

\begin{tabular}{lccc} 
Régions & $\begin{array}{c}\text { Nb. de porcs } \\
\text { examinés }\end{array}$ & $\begin{array}{c}\text { Nb. de porcs } \\
\text { ladres }\end{array}$ & $\begin{array}{c}\text { Prévalence } \\
\mathbf{( \% )}\end{array}$ \\
\hline $\begin{array}{l}\text { Mayo-Danay } \\
\text { (Cameroun) }\end{array}$ & 441 & 68 & 15,4 \\
$\begin{array}{l}\text { Mayo-Kebbi } \\
\text { (Tchad) }\end{array}$ & 411 & 107 & 26,0 \\
\hline
\end{tabular}

* 155 et 20 respectivement chez des porcs adultes $(n=566)$ et jeunes $(n=286)$

\section{Tableau V}

Prévalence de la cysticercose porcine chez les porcs abattus (inspection de viande)

\begin{tabular}{|lccc|}
\hline & $\begin{array}{c}\text { Nb. total de } \\
\text { porcs inspectés }\end{array}$ & $\begin{array}{c}\text { Nb. de porcs } \\
\text { infestés }\end{array}$ & $\begin{array}{c}\text { Prévalence } \\
(\%)\end{array}$ \\
\hline Porcs adultes & 23 & 7 & 30,4 \\
Porcs jeunes & 28 & 1 & 3,6 \\
\hline
\end{tabular}


certaine amélioration du système de l'élevage, ont montré qu'entre 1997 et 2000 la prévalence de la cysticercose porcine a sensiblement diminué dans la province de l'Ouest (respectivement 2,3 et 6,1 p. 100).

Les résultats du test Elisa pour la détection d'antigènes circulants (Ag-Elisa) (tableau VI) réalisé sur 264 porcs apparemment non infectés à l'examen clinique ont montré que 38,9 et 40,8 p. 100 étaient positifs respectivement dans le Mayo-Danay et le MayoKebbi. D'après Brandt et coll. (3), cela indiquait que ces animaux étaient des porteurs de cysticerques vivants, parce que l'Ag-Elisa ne détecte pas des cysticerques morts. Ce test a une sensibilité de 84,6 p. 100 et une spécificité de 99,1 p. 100 (12). Bien que la divagation des porcs et la défécation en dehors des latrines ait été nettement plus fréquentes au Mayo-Kebbi qu'au Mayo-Danay et que, par conséquent, le taux de positifs au langueyage ait été plus élevé dans la première région (26 p. 100 au Mayo-Kebbi contre 15,4 p. 100 au Mayo-Danay), les séroprévalences observées dans les deux régions

\section{Tableau VI}

Séroprévalence de la cysticercose porcine (Ag-Elisa)

\begin{tabular}{lccc} 
Régions & $\begin{array}{c}\text { Nb. de sérums } \\
\text { examinés }\end{array}$ & $\begin{array}{c}\text { Nb. de sérums } \\
\text { positifs }\end{array}$ & $\begin{array}{c}\text { Séroprévalence } \\
\text { (\%) }\end{array}$ \\
\hline $\begin{array}{l}\text { Mayo-Danay } \\
\text { (Cameroun) }\end{array}$ & $139 *$ & $54^{* *}$ & 38,9 \\
$\begin{array}{l}\text { Mayo-Kebbi } \\
\text { (Tchad) }\end{array}$ & 125 & 51 & 40,8 \\
\end{tabular}

* 9 sérums sur 102 du Mayo-Danay ont été prélevés sur les porcs abattus, apparemment non infestés à l'inspection ante et post mortem

** 2 sérums sur 9 ont réagi positivement à l'analyse sérologique

\section{BIBLIOGRAPHIE}

1. AWA D.N., NJOYA A., NGO TAMA A.C., EKUE F.N., 1999. The health status of pigs in North Cameroon. Revue Elev. Méd. vét. Pays trop., 52: 93-98.

2. BOA M.E., BOGH H.O., KASSUKU A.A., NANSEN P., 1995. The prevalence of Taenia solium metacestode in pigs in northern Tanzania. J. Helminthol., 69: 113-117.

3. BRANDT J., GEERTS S., DE DEKEN R., KUMAR V., CEULEMANS F., BRIJS L., FALLA N., 1992. A monoclonal antibody based ELISA for the detection of circulating excretory-secretory antigens in Taenia saginata cysticercosis. Int. J. Parasitol., 22: 471-477.

4. CHAMBERS P.G., 1987. Carcass and offal condemnation of meat inspection in Zimbabwe. Vet. J., 1: 11-18.

5. DORNY P., VERCAMMEN F., BRANDT J., VANSTEENKISTE W., BERKVENS D., GEERTS S., 2000. Sero-epidemiological study of Taenia saginata cysticercosis in Belgian cattle. Vet. Parasitol., 88: 43-49.

6. GEERTS S., 1993. The taeniasis-cysticercosis complex in Africa. Bull. Séances Acad. R. Sci. Outre-Mer, 38: 245-264.

7. GEERTS S., 1995. Cysticercosis in Africa. Parasitol. Today, 11: 389.

8. GRABER M., CHAillouX A., 1970. Existence au Tchad de la ladrerie porcine à Cysticercus cellulosae (Rudolphi). Revue Elev. Méd. vét. Pays trop., $23:$ 49-55.

9. MARTY P., HERZOG U., MARTY-JAUSSAN I., LE FICHOUX Y., DOUCET J., 1985. Deux cas de cysticercose observés au Cameroun. Méd. trop., 45 : 83-86.

10. MARTY P., MARY C., PAGLIARDINI G., QUILICI M., LE FICHOUX Y., 1986. Courte enquête sur la cysticercose et la taeniasis à Taenia solium dans un village de l'Ouest-Cameroun. Méd. trop., 46 : 181-183.

11. NANA-NUKECHAP M.F., GIBBS E.P.J., 1985. Socio-economic effects of African Swine Fever in Cameroon. Trop. Anim. Health Prod., 17: 183-184. ont été comparables. Ceci pourrait s'expliquer par le fait que l'AgElisa détecte aussi bien les infestations légères que les massives, tandis que le langueyage détecte plus facilement les infestations massives. On peut donc supposer que le nombre de porcs faiblement positifs a été plus important au Mayo-Danay qu'au Mayo-Kebbi parce que, dans la première région, la divagation des porcs a été moins fréquente et, par conséquent, l'accès aux proglottis entiers de $T$. solium qui est à la base d'infestations massives.

\section{CONCLUSION}

Cette étude ainsi que celle de Njoya et coll. (13) menée dans la région de Garoua (province du Nord) indiquent que le Nord Cameroun et le sud-ouest du Tchad sont des régions hyperendémiques pour la cysticercose porcine. Cela s'explique par le fait que les conditions d'infestation des porcs avec les œufs de T. solium et des hommes par les métacestodes sont réunies dans ces régions. En effet, l'inexistence ou la rareté des latrines, même dans les centres urbains, et les défécations à l'air libre, la divagation permanente ou semi-permanente des porcs, les abattages des porcs qui se font en général à domicile et/ou dans des endroits inaccessibles aux services vétérinaires, l'inspection quasi inexistante de la viande de porcs et enfin la méconnaissance des aspects zoonotiques du parasite constituent des conditions idéales pour l'accomplissement du cycle biologique du parasite et la perpétuation de la zoonose dans les deux régions.

\section{Remerciements}

Cette étude a été faite avec le support financier de la Direction générale de la Coopération internationale, Bruxelles (Accord cadre avec l'Institut de médecine tropicale, Anvers).

12. NGUEKAM, 1998. La cysticercose porcine dans les départements de la Mifi et des Bamboutos. Thèse Master Sci., Institut de médecine tropicale, Anvers, Belgique, $41 \mathrm{p}$.

13. NJOYA A., AWA D.N., MOUSSA C., NGO TAMA A.C., CARDINALE E., EBANGI L., NGUANGUE J.M., 1996. L'élevage porcin au Nord-Cameroun : situation actuelle et possibilité d'amélioration. Rapport technique. Garoua, Cameroun, Irad, 51 p.

14. ONAH D.N., CHIEJINA S.N., 1995. Taenia solium cysticercosis and human taeniasis in the Nsukka area of Enugu State, Nigeria. Ann. trop. Med. Parasitol., 89: 399-407.

15. POUEDET S., 2001. La cysticercose porcine dans le Menoua (Ouest Cameroun). Thèse Master Sci., Institut de médecine tropicale, Anvers, Belgique, $41 \mathrm{p}$.

16. PREUX P.M., MELAKU Z., DRUET-CABANAC M., AVODE G., GRUNITZKY E.K., BOUTEILLE B., CRUZ M., DUMAS M., 1996. Cysticercosis and neurocysticercosis in Africa: Current status. Neurol. Inf. Epidemiol., 1: 63-68.

17. TSANG V.C., WILSON M., 1995. Taenia solium cysticercosis: An under recognized but serious public health problem. Parasitol. Today, 11: $124-126$.

18. ZOLI A., GEERTS S., VERVOORT T., 1987. An important focus of porcine and human cysticercosis in West Cameroon. In: Geerts S., Kumar V., Brandt J., Eds, Helminth zoonoses. Dordrecht, Netherlands, Martinus Nijhoff, p. 85-91.

19. ZOLI A., NGUEKAM J.P., DORNY P., GEERTS S., BRANDT J.R.A., 1998. Taenia solium cysticercosis in West Cameroon. In: Mukaratirwa S., Obwolo M.J., Eds, Livestock production for rural development. Proc. IXth Int. Conf. Association of Institutions of Tropical Veterinary Medicine, Harare, Zimbabwe, 14-18 September 1998, p. 571-577.

Reçu le 01.08.2001, accepté le 18.02.2002 


\section{Summary}

Assana E., Zoli P.A., Sadou H.A., Nguekam, Vondou L., Pouedet M.S.R., Dorny P., Brandt J., Geerts S. Prevalence of Porcine Cysticercosis in Mayo-Danay (North Cameroon) and Mayo-Kebbi (Southwest Chad)

A study was conducted from May to October 1999 in the Mayo-Danay Department (far north of Cameroon) and MayoKebbi District (southwest of Chad) to determine the prevalence of porcine cysticercosis and identify its main contributing factors. Hygienic conditions, where the population lived and pigs were reared, were very poor and contributed to heavy porcine infections by Taenia solium cysticerci. Out of 126 households visited, $42 \%$ did not have latrines. Pigs roaming freely or semi-freely were thus exposed to human feces laid around the habitations. The tongue inspection method to establish a clinical diagnosis showed that $20.5 \%$ of the 852 pigs harbored cysticerci. Carcass inspection at local slaughterhouses revealed that $15.7 \%$ of the 51 slaughtered pigs were infected. Out of 264 pig sera tested by ELISA for cysticercus circulating antigen detection, 105 $(39.8 \%)$ were positive. The results showed that Mayo-Danay and Mayo-Kebbi were major foci of Taenia solium porcine cysticercosis.

Key words: Swine - Taenia solium - Cysticercosis - Cameroon Chad.

\section{Resumen}

Assana E., Zoli P.A., Sadou H.A., Nguekam, Vondou L., Pouedet M.S.R., Dorny P., Brandt J., Geerts S. Prevalencia de la cisticercosis porcina en el Mayo-Danay (norte del Camerún) y el Mayo-Kebbi (sudoeste del Chad)

La finalidad de un estudio llevado a cabo entre mayo y octubre de 1999, en el departamento de Mayo-Danay (extremo norte del Camerún) y en la prefectura de MayoKebbi (sudoeste del Chad), fue la de determinar la prevalencia de la cisticercosis porcina y la de identificar los principales factores que la favorecen. Se mostró que las condiciones higiénicas en las cuales vive la población, así como las de la producción de cerdos fueron muy mediocres, con consecuencias como infestaciones masivas de la población porcina por cisticercos de Taenia solium. En efecto, $42 \%$ de las 126 explotaciones visitadas no poseían letrinas y los cerdos, vagando semi permanente o permanentemente, tenían acceso fácil a las materias fecales humanas dispersas en los alrededores de las casas. El diagnóstico clínico realizado mediante el método lingual (langueyage) mostró que 20,5\% de los 852 cerdos vivos examinados eran portadores de cisticercos. La inspección de las carcasas, llevada a cabo en los mataderos locales, reveló que $15,7 \%$ de los 51 cerdos sacrificados fueron granujientos. De los 264 sueros de cerdos sometidos al test Elisa para la detección de antígenos circulantes de cisticercos, 105 (39,8\%) fueron positivos. Estos resultados indican que Mayo-Danay y Mayo-Kebbi son focos importantes de cisticercosis porcina por Taenia solium.

Palabras clave: Cerdo - Taenia solium - Cisticercosis Camerún - Chad. 